\title{
$¿$ ¿e qué modo las instituciones públicas deberían acomodar a los inmigrantes? Un marco analítico para el análisis *
}

\author{
Ricard Zapata-Barrero **
}

\section{El problema: la relación entre el inmigrante y las instituciones públicas}

El principal objetivo de este artículo es ofrecer una vía para analizar el fenómeno de la inmigración desde una perspectiva politológica. Lo que distingue la ciencia política de las demás disciplinas científicas sociales que tratan también esta cuestión es que aborda el tema en términos de relación entre el inmigrante $y$ las instituciones públicas ${ }^{1}$. Le interesan sobre todo las relaciones verticales y no tanto las horizontales. Esto es, su preocupación básica es analizar cómo afecta la presencia de los inmigrantes a nuestro sistema político, a nuestra tradición liberal y democrática (R. ZAPATA-BARRERO, 2000c), a nuestro comportamiento y cultura políticas, y no tanto el impacto que tiene la inmigración sobre nuestras estructuras sociales. Esta relación entre inmigrante e instituciones públicas limita un contexto que denomino como esfera pública. El enfoque politológico que propongo parte de la convicción de que la primera prueba de realidad que tiene el inmigrante cuando llega a nuestro país (nuestra Comunidad Autónoma, nuestra ciudad) es resultado de sus primeras relaciones con las instituciones públicas. Podría trazarse la biografía de un inmigrante describiendo hasta qué punto sus expectativas de vida son moldeadas por sus relaciones con la estructura básica institucional del Estado (de la Comunidad Autónoma, de la ciudad) donde ha decidido instalarse.
Este enfoque tiene también como premisa el hecho de que al abordar el tema de las políticas de inmigración existen dos niveles de análisis, que si bien están interconectados, analíticamente siguen diferentes lógicas de argumentación y plantean diferentes preguntas (R. ZaPATA-BarRero, 2000d, e) ${ }^{2}$. En un primer nivel están las políticas de acceso a nuestro territorio; en un segundo nivel, las políticas de coexistencia, una vez el inmigrante ha sido admitido.

En el nivel de acceso prima la lógica entre fronteras abiertas y cerradas. Esto es, los debates suelen tomar una posición entre estos dos extremos. Los argumentos que se esgrimen responden a dos tipos de preguntas, una que incide en la cualidad y otra en la cantidad de las personas «autorizadas» a entrar legalmente: la pregunta sobre quiénes entran y la pregunta sobre cuántos entran. En la primera, las respuestas suelen ser políticas; en la segunda, priman más factores macrocoyunturales (mercado, demografía, bienestar, etc.). Las decisiones políticas sobre el quiénes suelen seguir criterios económicos, de procedencia nacional, de afinidad cultural, de proximidad geográfica, «deudas históricas» con antiguas colonias, también entran aquí los convenios bilaterales de España y países emisores. En la segunda pregunta, esto es, el cuántos, las respuestas giran en torno a los criterios de las políticas de cupos. El tema central del debate es que las decisiones no son solamente políticas (como en el primer caso), sino más bien de mercado. Priman, así, criterios macrocoyunturales económicos, demográficos, nivel de bienestar, sistema de libertades, en definitiva, el debate sobre los límites de la tolerancia. 
En el segundo nivel de análisis, el de las políticas de coexistencia, la pregunta pasa a ser cómo. Aquí prima la lógica entre la inclusión y la exclusión. Esto es, las discusiones apuntan a cómo conseguir el máximo de inclusión en la corriente principal de la sociedad de aquellos que están inicialmente excluidos por decisión exclusiva del Estado. Aquí es donde entra el debate sobre la integración (acomodación) de los inmigrantes. Se asume que toda política de inmigración tiene unos efectos y unas consecuencias sobre la estructura política de nuestras sociedades. El debate también gira en torno a qué criterios debemos considerar como legítimos desde un punto de vista democrático y liberal para dar acceso (incluir) a los inmigrantes en nuestras principales esferas públicas.

El objetivo de este artículo es ofrecer un marco analítico para analizar este segundo nivel de las políticas de inmigración, a saber, el de la coexistencia ${ }^{3}$. Dicho marco es el resultado de una investigación empírica realizada a lo largo del año 1999 en la ciudad de Barcelona, donde entrevisté a 53 actores procedentes de las instituciones y del mundo asociativo, comprometidos con la acomodación de los inmigrantes ${ }^{4}$.

Articularé esta propuesta en cuatro pasos. En la sección I detallo las premisas básicas para abrir esta vía de análisis, consistente en defender una concepción compleja de la acomodación. En la sección II, la razón de ser de este artículo, propongo un marco analítico, estructurado en dos apartados: sus límites externos (apartado 1) e internos (apartado 2). Por último, en la sección III, enumero algunos contextos donde podría aplicarse el marco en futuras investigaciones.

\section{Una concepción compleja de la acomodación}

El fenómeno de la inmigración plantea más preguntas que respuestas. Los actores más directamente comprometidos con la acomodación de los inmigrantes tienen una sensación generalizada de que sus prácticas han cerrado un círculo. Sus reuniones periódicas para planificar acciones de carácter reivindicativo o de gestión de los conflictos expresan cada vez más un convencimiento de estar encerrados en una lógica viciosa, donde se comparte mejor «lo que no debe hacerse» que «lo que debe hacerse». Faltan no sólo incentivos y contactos con otras experiencias, sino también un marco de referencia que permita analizar el sistema de supuestos y las lógicas de acción de los actores para volver a orientar de forma constructiva sus discursos y actuaciones. Esta desorientación práctica está en relación con cierto confusionismo teórico que existe en el debate actual. En nuestros días, el debate en torno a la aco- modación de los inmigrantes asume como idea recibida que el producto final de su análisis podrá ser aplicado, o bien solamente en una esfera de la estructura básica de la sociedad, o bien en todas las esferas sin distinciones. La literatura existente suele ser, por lo tanto, parcial con un carácter universalista.

Este hecho manifiesta que existe una tendencia a seguir dos tipos de enfoques que tienen en común abordar el problema de la acomodación de forma simple y no compleja: unos enfoques universalistas y otros parciales. El problema es que se ignoran mutuamente. Los enfoques universalistas hacen propuestas de políticas de acomodación susceptibles de ser aplicadas en cualquier sector público, ignorando las particularidades de cada esfera. Los enfoques parciales hacen lo contrario. Se centran en políticas de acomodación en una esfera determinada (vivienda, educación, etc.), pero pierden visión global de conjunto, en cómo podrían afectar sus propuestas a otros sectores públicos. No tienen sensibilidad transversal, relacional entre esferas.

Cada uno de estos problemas nos invita a abordar el tema de la acomodación de los inmigrantes de una forma compleja, conectando los enfoques universalistas y parciales. Ésta es la intención básica del marco analítico que proponemos. Pretende ser útil como sistema de referencia susceptible de abrirnos una vía para el análisis que nos permita discriminar entre diferentes posibles políticas de acomodación según las esferas. Antes de presentarlo, déjenme introducir los tres puntos focales que me han ayudado a construirlo. A saber, el vínculo entre dimensión normativa e institucional (primer punto focal) el objetivo de toda política de acomodación (segundo punto focal) y, finalmente, el vínculo entre dos ámbitos de análisis, el de la teoría política y el de las políticas públicas (tercer punto focal).

El primer punto focal vincula dos dimensiones: una dimensión normativa y otra institucional. La primera tiene como centro el concepto mismo de «acomodación». Traza tres modelos susceptibles de ser aplicados empíricamente para describir el modo en que una política pública acomoda a los inmigrantes siguiendo determinadas lógicas de acción. La dimensión institucional está compuesta por dos centros con características diferentes: al primero le damos un carácter cambiante y subjetivo, el de los actores implicados en la acomodación (asociaciones en general, tanto de inmigrantes como ONGs, partidos políticos, organizaciones de interés, y unidades administrativas públicas); el segundo tiene un carácter estructural y objetivo, el de las diferentes esferas institucionales (convivencia social, empadronamiento, vivienda, trabajo, sanidad, educación, seguridad y cultura).

En segundo lugar, partimos de la premisa que en el momento en que un inmigrante llega en un país su espacio de acción se ve inmediatamente circunscrito por la estructura básica institucional del Estado (de la Comunidad Autónoma, de la ciu- 
dad) que le acoge. Esto es, los inmigrantes, «cuando llegan», carecen de los bienes que las instituciones distribuyen a sus ciudadanos. La intención básica de una política de acomodación es que se beneficien también de esta distribución. El problema reside, y aquí entramos en uno de los temas centrales, en que las instituciones públicas encargadas de dicha distribución suponen que se encuentran con unos potenciales receptores con iguales propiedades que los autóctonos, y al mismo tiempo que aplican sus políticas dificultan que el inmigrante mantenga su propio estilo de vida de forma autónoma. Parto, así, de la premisa de que el objetivo principal de una política de acomodación es dotar a los inmigrantes de autonomía al actuar en la esfera públi$\mathrm{ca}^{5}$. Aunque luego lo ampliaré con más detenimiento, «acomodación» significa (y perdonen por la tautología) «sentirse cómodo al actuar en la esfera pública». Esto es, que el inmigrante no tenga la necesidad de justificar los conflictos que tiene al rela. cionarse con las diferentes esferas de las instituciones públicas por su situación jurídica, su procedencia nacional y su cultura. La familia semántica de estar cómodo quizá ampliará lo que estoy diciendo. «Sentirse cómodo» es: sentir descanso físico y psíquico, sin esfuerzo ni molestia excesivas, confortable, holgado, a gusto. En definitiva, sentirse bien consigo mismo y con la sociedad, sin sentimientos de culpa ni rencor. También tiene el sentido de que los intereses subjetivos de una persona, aunque no sean realizables, tienen opción para ello. A veces no nos sentimos cómodos cuando se produce un intervalo entre nuestros intereses y la posibilidad práctica de poder realizarlos con los recursos de que disponemos. Salvando distancias, concibo las políticas de acomodación muy similares a las políticas dirigidas a los minusválidos. Se adaptan aceras, entradas en autobuses, etc., con la finalidad de que puedan valerse por sí mismos y sentirse «cómodos» en la esfera pública, es decir, autónomos. Más adelante ampliaré este concepto central.

El tercer punto focal reside en que la tensión básica para diseñar una política de acomodación puede localizarse en dos niveles: uno perteneciente a la política pública y el otro al ámbito de la teoría política. Desde el punto de vista de la política pública, la tensión existente entre unas políticas especificas y unas politicas genéricas. Las primeras tendrían como principales receptores únicamente a los inmigrantes en virtud de sus propiedades específicas. Las políticas genéricas (también mal llamadas «normalizadas») tratarían a los inmigrantes como al resto de la población autóctona, sin considerar como criterios de orientación sus propiedades distintivas. Entre ambos extremos existen lo que denominaremos políticas genéricas de acción especifica. Estas políticas incorporarían dentro de una lógica genérica la especificidad de los inmigrantes, para poder incluirlos como potenciales receptores y evitar, así, los efectos excluyentes secundarios de las políticas específicas. Las justificaciones que se dan para defender una opción frente a las otras son complejas (R. ZAPATA-BARRERO, 2001d). Lo que me interesa aquí avanzar es que propongo analizar estas tres opciones políticas de aco- modación desde el punto de vista de la teoría política $y^{y}$ no estrictamente de la política pública. Esto significa, y aquí me adentro en el segundo nivel de análisis, el que particularmente profundizaremos, que conduciremos los argumentos que se esgriman en el primer nivel hacia este segundo.

Desde el punto de vista de la teoría política, la tensión estriba en si la estructura básica de la sociedad debe variar para acomodar a los inmigrantes, ya que la actual ha estado concebida para una población predeterminada con características similares y, por lo tanto, de acuerdo con las propiedades de los receptores, de forma simple y homogénea. Como tendré ocasión de ampliar más adelante, las justificaciones que se proporcionan para defender la necesidad de modificar o no la estructura básica son muy diversas. Esta controversia constituirá uno de los principales centros de atención del marco analítico. Las políticas genéricas puras abogarían por mantener la estructura actual, mientras que las políticas genéricas de acción específica o estrictamente específicas estarían orientadas por una preocupación de incorporar propiedades distintivas de los inmigrantes (lo que denominaré como los bienes mediadores de los inmigrantes) dentro de la estructura básica existente con su consecuente modificación.

A partir de estas primeras constataciones la pregunta básica que podemos plantearnos es la siguiente: ¿de qué modo las instituciones públicas deberían acomodar a los inmigrantes? Esta pregunta parte del supuesto de que la llegada de inmigrantes no provoca directamente conflicto, sino que es más bien la forma en que está estructurada institucionalmente la sociedad la que está en el origen de los conflictos. Por lo tanto, todos los actores comparten implícita o explícitamente que la inmigración afecta directamente la estructura básica institucional. El problema reside, y en este punto se sitúa la pregunta que planteamos, en el modo en que las instituciones existentes deben reorientarse para dar una respuesta adecuada al gestionar estas nuevas demandas. En esta dinámica es donde se sitúan tanto la desorientación práctica como la confusión teórica aludidas: no se tienen propuestas sólidas para orientar las modificaciones estructurales, y las únicas líneas teóricas para guiar estas propuestas prácticas son o bien universalistas o bien parciales, sin conectar precisamente estos dos enfoques.

Por último, el tema central de reflexión del marco analítico se construye conectando todos los puntos anteriores. Parte de la idea de que no existe una forma simple, uniforme, global y universal de acomodar a los inmigrantes, sino que la política de acomodación es compleja, específica de la esfera donde debe implementarse y de los bienes mediadores que los inmigrantes requieren para poder beneficiarse, sin perder su autonomía, de los bienes que se distribuyen. Se formula de la forma siguiente: no existe una única lógica de politica de acomodación, sino varias. Esta multiplicidad depende de la combinación del bien que 
se quiere asignar a la población inmigrante y de los bienes mediadores que dicha población requiere para poder ser autónomas.

\section{Propuesta de un marco analítico}

Esta parte consta de dos apartados: en el primero presento los límites externos del marco analítico y en el segundo su estructura y contenido.

\section{Límites externos del marco analítico}

Este primer capítulo está organizado en tres puntos. En el primero presento la base teórica del marco analítico, enfocada en la noción de Justicia Local; en el segundo preciso su base conceptual, centrada en la noción de acomodación; en el tercer punto me ocupo de su base normativa, fundada en tres modelos de acomodación política.

\subsection{Base teórica del marco analitico: Justicia Local e inmigración}

La base teórica del marco analítico combina tres tipos de teorías que habitualmente aparecen de forma separada. Por un lado, los trabajos de M. WALZER (1983) y de J. ElSTER (1990, 1994, ed. 1995); por otro lado, una literatura básica sobre acción colectiva y teoría de bienes (M. OLSON, 1965; J. ELSTER, 1985; J. Hovi, 1986; P. E. OlIver, 1993; J. Jordana) ${ }^{6}$; y, por último, teorias normativas de la democracia y de la justicia para completarlos (J. Rawls, 1971, 1993; A. Hamlin y Ph. PetTit, eds. 1989; M. WARREN, 1992; R. A. DAHL, 1992).

El uso que haré de toda esta literatura estará muy enfocado al objeto de estudio diseñado anteriormente. Aunque no es mi propósito presentar en detalle cada uno de estos trabajos, sí que es necesario precisar, al menos, que las líneas básicas iniciales del marco analítico están directamente inspiradas en la vía de análisis elaborada por ELSTER, basada a su vez en las Esferas de la Justicia de WALZER. Es, pues, legítimo que presente, dentro del entramado conceptual de ELSTER, aquellos aspectos que adaptaré a mi estudio, intentando no simplificarlo demasiado.

A través de Justicia Local ELSTER diseña un marco susceptible de poder describir las diferentes formas en que las instituciones asignan bienes escasos. Su argumento es que no existe una única forma de asignar bienes escasos, sino muchas, y que esta variedad depende mayoritariamente de la especificidad del bien que se quiere distribuir y de las propiedades de los potenciales beneficiarios. Su principal interés es que dicho marco sea útil para describir y explicar por qué las instituciones optan por unos principios de asignación en lugar de otros.

Basándome en estas ideas, por Justicia Local entenderé principalmente dos significados que se complementan. Por un lado, un marco de referencia que orienta políticas programadas por instituciones más o menos autónomas que, aunque pueden estar limitadas por normas dictadas por el gobierno estatal, tienen una cierta autonomía para diseñar e implementar el plan que prefieren (ELSTER, 1994: 16). Como consecuencia, los bienes que distribuyen dichas instituciones locales pertenecen a esferas públicas separadas del sistema de distribución global (ELSTER, 1990: 117). Por otro lado, que dichos bienes son asignados a los inmigrantes por diferentes procedimientos según la esfera competente. En este segundo sentido «local» se refiere a los diferentes sectores públicos que intervienen en el proceso de acomodación de los inmigrantes ?

Es cierto que el mismo ELSTER utiliza la política de inmigración como caso de estudio de su Justicia Local (1994), y algunos de sus seguidores la han aplicado en el contexto de EEUU (G. MACKIE, 1995). Pero la forma en que se aplica la teoría contrasta sustancialmente de la mía. Una comparación me ayudará a añadir más elementos para limitar la base teórica del marco analítico.

La diferencia más sustancial de la que se derivan las otras es que se utiliza la inmigración como un bien (escaso). Como consecuencia, una política de inmigración establecerá la forma en que ese bien es asignado a personas que lo solicitan. Su análisis se encuentra en el nivel de acceso y no de coexistencia, una vez los inmigrantes han sido aceptados. El marco analítico que propongo no considera la inmigración como un bien deseable para ciertas personas, sino como lo que ELSTER denomina «burden» que traduciremos descriptivamente como «carga». Esto implica que la inmigración será tratada como la principal receptora de bienes estructurales, siendo una carga que acota su espacio de acción, y cuyos límites no dependen directamente de su voluntad sino de la del gobierno ${ }^{8}$. Aunque reconocemos que la forma en que se distribuyen estos límites son determinantes, no nos centraremos directamente en esta cuestión, que nos obligaría a considerar la inmigración como un bien distribuible. El marco analítico se aplica una vez se ha producido el paso de la inmigración percibida como un bien a una carga. Como precisa ELSTER, la distinción entre «bien» y «carga» no es estricta. Una carga puede ser conceptuada como un bien, y viceversa (ELSTER, 1995: 5). Una carga, a diferencia de un bien, representa siempre una pérdida y no una ganancia. La inmigración como bien es una ganancia en comparación con las otras personas que se han quedado en la frontera terrestre o que han conseguido entrar por vías no reconocidas jurídi- 
camente. Pero también es una pérdida cuando, una vez adquirido ese bien, el referente comparativo se traslada automáticamente hacia la población autóctona, o hacia otros inmigrantes nacionalmente privilegiados por el principio de afinidad cultural.

En mi uso, pues, la inmigración siempre es considerada como un límite para la acción en la sociedad receptora, una falta de autonomía para la decisión. El análisis se hará, por lo tanto, tomando como punto focal al inmigrante y no a la sociedad receptora o a sus ciudadanos. Según esta óptica, la ciudadanía sí que será tratada como un bien, y no como una carga. Es un bien evidentemente para el inmigrante y, por lo tanto, como todo bien, es percibido si se adquiere como ganancia y no como pérdida. En este nivel de coexistencia, una vez la persona no nacional ha adquirido el bien de ser considerada como inmigrante legal, la política pasa de ser un asunto de Justicia Global a ser principalmente una cuestión de Justicia Local. Aquí es donde la política de acomodación juega un papel fundamental, diría vital desde el punto de vista del inmigrante, en tanto que se pasa de la percepción de la inmigración como un bien a una carga ${ }^{9}$. Se sigue, pues, que los receptores de una política de acomodación son los inmigrantes y no los ciudadanos, y los bienes que requieren beneficiarse para llevar a cabo la acomodación no son siempre escasos sino también abundantes. Entran aquí en juego no solamente políticas de distribución, sino también regulativas ${ }^{10}$.

Creo que es el momento de aclarar uno de los conceptos que recorren estas páginas desde el principio y que constituye el pilar conceptual básico del marco analítico: a saber, la noción de acomodación. El uso de este término no responde a razones retóricas o simplemente de comodidad analítica, dada la sobrecarga y uso indiscriminado del término integración, sino más bien a razones metodológicas que enseguida justificaré ${ }^{11}$.

\subsection{Base conceptual del marco analítico: la acomodación de los inmigrantes}

Según la definición dada por María MOLINER, «acomodar» significa, por un lado, poner cosas o personas en el sitio o la postura conveniente, o de modo que quepan en cierto espacio; por otro lado, hacer que dos cosas (objetos, personas, doctrinas, teorias, por ejemplo) sean compatibles y no estén en oposición, hacer que un objeto se ajuste a la forma o tamaño de otro. Su forma reflexiva («acomodarse») tiene a veces el sentido de arreglarse para vivir con los medios de que se dispone, aunque sean escasos. Moliner contempla como pertenecientes a la familia semántica de «acomodar» y «acomodarse» los términos siguientes: adaptar, ajustar, amoldar, acondicionar, acoplar, adecuar, armonizar, cuadrar, encajar, juntar, pegar, colocar bien, sincronizar, ponerse de acuerdo, avenirse, coordinar, entre otros. «Acomodación» significaría, en este caso, la acción de acomodar. Palabras que utilizan la misma raíz y que precisan el sentido es, evidentemente, la figura ya olvidada del «acomodador» del cine o teatro; la persona que nos acompañaba, tras comprobar nuestra entrada (nuestro «permiso de residir temporalmente» en el cine o teatro), hasta el lugar que nos correspondía (si estaban numeradas) o el lugar vacante (si no estaban numeradas). Este sentido podría muy bien servir de imagen cuando el «acomodador» es el poder político, y el espacio no es el cine o teatro sino la ciudad, la Comunidad Autónoma o el Estado en general. En este sentido, una política de acomodación significaría una política de acomodar a los inmigrantes que ya tienen «entrada». La diferencia con la imagen es que el acomodador y los que deciden los criterios a seguir para llegar a cabo la acomodación coinciden. Correspondería en este caso a la administración pública. Aunque también, como en el ejemplo del acomodador del cine, su poder de decisión está limitado por instituciones jerárquicamente superiores (el director de la compañía del cine, por ejemplo, o, en nuestro caso, la Administración central).

El término en todo caso es descriptivo. Etimológicamente está compuesto de tres partículas «a-co-modar». El prefijo «a-» se usa habitualmente, aparte de para negar aquello que la palabra expresa, que no sería este caso, para formar a partir de un nombre o adjetivo, un verbo, transformando en acción el significado de aquéllos (ejemplos: amontonar, abreviar). En este mismo sentido, significa también «poner» lo que la palabra expresa (ejemplos: abanderar, anotar), otras veces «dar la forma» de lo que el adjetivo o nombre significa (ejemplos: ahorquillar, abarquillar). «Comodar» viene del adjetivo «cómodo» que significa etimológicamente «commodus», el cual está compuesto de con-modus. Significa que una cosa es manejable o realizable con facilidad, sin molestias físicas (ni psíquicas).

El término «integración», por el contrario, tiene un uso más normativo que descriptivo. Además, está claro que actualmente el término se extiende demasiado y como consecuencia se desvanece su núcleo conceptual: si todo es integración, entonces nada lo es en realidad, y en definitiva termina por ser analíticamente carente de sentido. Éste no es el lugar adecuado de recorrer su biografía científica social y entrar en discusiones nominalistas ${ }^{12}$. En términos conceptuales, integración es un término «esencialmente polémico», en tanto que sería una empresa difícil separar su núcleo conceptual de las concepciones que implica. Según María Moliner, «integración» es la acción de «integrar», que significa hacer o contribuir a formar un todo o conjunto con partes diversas (pensemos, por ejemplo, en la «integración europea»), hacer entrar una cosa (el inmigrante, en nuestro caso) en otra más amplia (la sociedad receptora, por seguir el ejemplo).

Según los usos que hemos dado, «acomodar» e «integrar» son términos relacionales, pero que difieren semánticamente. 
Desde la óptica teórica del marco analítico, «acomodar» no implica necesariamente que el inmigrante pierda su autonomía al relacionarse con la estructura básica de la sociedad, sino que simplemente varían ciertas de sus características, por voluntad propia, con la finalidad de «encajar en su nuevo contexto», teniendo que experimentar un proceso similar la estructura básica. Sin embargo, «integrar» significa que el inmigrante pierde cierta independencia al formar parte de la sociedad receptora (pensemos de nuevo en la integración europea y los debates en torno a la pérdida de soberanía estatales). Y esta pérdida, lo queramos o no, invita a la argumentación normativa, a la formación de concepciones. Cabe añadir que en ambos términos relacionales el sujeto (el inmigrante) tiene un carácter dinámico, mientras que el objeto (la sociedad receptora) es estático, se mantiene prácticamente igual. Veremos a lo largo de este estudio que el marco analítico nos permitirá poner en duda este uso común. En algunos resultados de las entrevistas se enfatiza, por ejemplo, que una política de acomodación bien hecha es aquella que tiene como objeto prioritario la acomodación del ciudadano al inmigrante, y no al revés. Es decir, se sugiere el fomento de una cierta cultura de recepción. Una de las ideas fuerzas que trataremos de seguir es que una política de acomodación implica que ambos componentes de la relación varien algunas de las propiedades de su situación inicial, esto es, la situación previa a la coexistencia.

\subsection{Base normativa del marco analítico: tres modelos de acomodación politica}

Utilizando como indicadores la participación en la decisión y gestión política de la diferencia cultural, y el carácter cultural de las diferentes esferas públicas, pueden precisarse tres modelos básicos de acomodación. Respectivamente, una exigencia de asimilación, de integración o de autonomía. Me interesa por el momento adentrarme en los fundamentos que legitiman cada una de estas políticas de acomodación ${ }^{13}$.

En el modelo asimilacionista no se expresa ninguna necesidad de variar los límites y el contenido actual de los valores que componen las diferentes esferas estructurales, eminentemente monoculturales o culturales autóctonas. Evidentemente, tampoco facilita la participación de los inmigrantes en las decisiones y gestión política de la diferencia, en manos exclusivamente de los ciudadanos. La coexistencia entre inmigrantes y autóctonos es percibida como competencia y, como tal, el grupo que dispone de más recursos para su supervivencia (el de los ciudadanos) podrá exigir a los otros las condiciones de acceso en las esferas públicas controladas por ellos mismos. En los términos de los dos indicadores, la asimilación puede definirse como decisión y gestión política de la diferencia en manos de la ciudadanía, y carácter monocultural de las esferas estructurales. El inmigrante que quiera acceder a ella deberá, por lo tanto, dejar para la esfera privada aquellas prácticas susceptibles de poner en tensión las ya establecidas. En términos políticos públicos su forma pura se inclina por políticas genéricas; en términos teórico-políticos, no hay variación de estructura básica. Resultado: el inmigrante tan sólo tiene su esfera privada para expresar su autonomía. En las diferentes esferas públicas no es autónomo, no puede expresar «con comodidad» algunos de sus bienes mediadores. Este modelo sólo defiende la intervención estatal cuando la cultura autóctona es vista como amenazada por cualquier forma de «invasor cultural». Podríamos decir que este modelo es el resultado de un dejar hacer cultural ${ }^{14}$, quien actúa como fundamento. Su preocupación básica es la conservación de la identidad autóctona, y su concepto preferido, cuando trata de temas de pluralismo cultural, es el de tolerancia.

El modelo integracionista compartiría con la orientación anterior la defensa de que la decisión y gestión política de la diferencia permanezca en manos de la ciudadanía, aunque admitiría algunas variaciones del contenido y de los límites culturales de las esferas públicas para permitir que ciertas prácticas de los inmigrantes tengan algún espacio. Independientemente del nivel donde se tomen las decisiones, se parte de la premisa de que ante la pregunta ćquién decide, quién controla los límites y el contenido de las esferas públicas?, la respuesta será siempre la misma: el ciudadano. Se sigue que su centro de atención son las diferentes esferas públicas. Este reconocimiento institucional tiene como impulso el deseo de apaciguar el conflicto emergente entre la población autóctona y la inmigrante, y obtener así un cierto grado de estabilidad y cohesión social. Sus conceptos preferidos al abordar el tema del pluralismo cultural son los de interculturalidad y respeto. En este caso, las esferas públicas podrían considerarse como interculturales, aunque este contenido y sus límites todavía estén controlados por la ciudadanía. Las demandas de los inmigrantes que consigan pasar el «filtro» del control ciudadano podrán así ser públicamente practicadas y reconocidas en las diferentes esferas locales ${ }^{15}$. La ciudadanía, no obstante, es quien en última instancia decide o no satisfacer dichas necesidades específicas de los inmigrantes. En contraste con la orientación anterior, la integracionista rechazaría la asimilación, pero exigiría a los extranjeros una integración. «Integración» porque, según el modelo, la ciudadanía estaría dispuesta a cambiar el carácter de sus esferas estructurales, «inter-culturalizarlas», si se me permite la expresión, pero sin posibilidad de participación política directa en la gestión de la diferencia por parte de los inmigrantes. En definitiva, las decisiones políticas todavía permanecerían exclusivamente en manos de los ciudadanos. En términos políticos públicos abogaría por políticas genéricas de acción específica (como, por ejemplo, creación de consejos de inmigrantes estrictamente consultivos y no vinculantes); en tér- 
minos teórico-políticos, apoyaría un cambio débil de la estructura básica.

El modelo autonómico compartiría con el integracionista la necesidad de crear esferas públicas sensibles a las prácticas de los inmigrantes. En tal caso su exigencia sería de integración. Pero en contraste con las dos anteriores orientaciones, estaría dispuesta a que los mismos inmigrantes residentes pudieran también formar parte del grupo decisor, o cuanto menos como gestor de la diferencia. Su centro de atención es que los inmigrantes tengan también medios de control de las decisiones sobre cómo gestionar las diferencias culturales. En este sentido, por su carácter participativo, este modelo fomentaría la autonomía plena de los inmigrantes. En una palabra, podrían participar en la toma de decisiones y en la gestión del contenido y de los límites de las diferentes esferas públicas, como un ciudadano más. Su preocupación básica es mantener una representatividad social y política del pluralismo cultural (no es ni la conservación de la identidad autóctona, ni la estabilidad), su concepto preferido es el de autonomía. En términos políticopúblicos abogaría más por políticas específicas; en términos teórico-políticos por una modificación fuerte de la estructura básica. Este modelo se encuentra, no obstante, ante un dilema que tendremos ocasión de recoger en el marco analítico más adelante: o bien seguir principios individuales o bien de grupo, o bien apoyar una autonomía individual, o bien respaldar una autonomía colectiva.

En resumen, y en nuestros términos analíticos, el modelo asimilacionista no reacciona ante el hecho de la inmigración proclamando una modificación de la estructura básica, sino todo lo contrario. El inmigrante, si quiere acomodarse, deberá aceptar los valores que sustentan la estructura institucional, y dejar los suyos propios para su ámbito privado. Por el contrario, tanto el modelo integracionista como el autonómico, aunque con diferentes intensidades y procedimientos, defienden la necesidad de modificar algunos aspectos de la estructura básica, con la finalidad de incorporar los nuevos bienes mediadores que requieren los inmigrantes para ser autónomos. La diferencia radica en los procedimientos: el modelo integracionista dejaría a los autóctonos la autonomía de decidir los criterios y el contenido de las esferas públicas pluriculturales, abogando mayoritariamente por políticas genéricas de acción especifica; mientras que el modelo autonómico dotaría a los mismos inmigrantes la posibilidad de decidir y de gestionar, junto con los ciudadanos, las esferas públicas, inclinándose más bien por políticas específicas.

\section{Limites internos: estructura y contenido del marco analítico}

Esquemáticamente el marco analítico puede representarse de la forma siguiente:

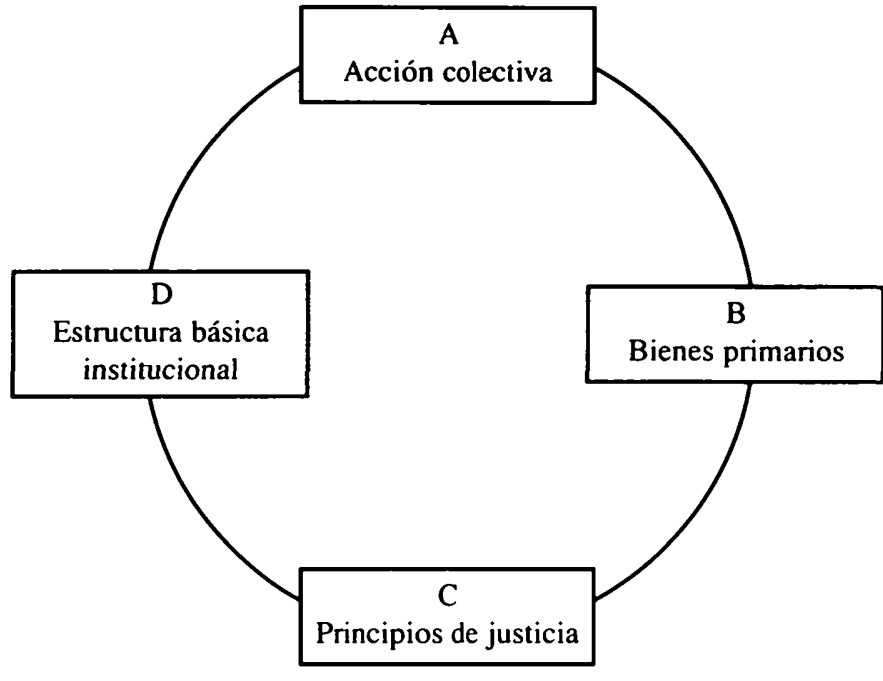

Nuestra premisa es que en todo proceso de diseño de una política de acomodación intervienen estos cuatro componentes. La primera característica de este marco es el carácter de sistema que tiene. De hecho, de aquí en adelante preferiremos hablar de sistema político de acomodación. Esto implica de entrada que cada uno de los componentes tiene una función mediadora. Por ejemplo, no puede entenderse la relación entre acción colectiva y principios de justicia si no es a través de otros mediadores: la estructura básica institucional y/o los bienes que se asignan; la relación entre los bienes y la estructura básica institucional sólo puede establecerse a través de los mediadores de la acción colectiva y/o principios de justicia, y así sucesivamente. Pero lo importante es detallar cada uno de ellos. Como punto de partida tomaremos definiciones simples.

\section{A. Acción colectiva}

Según la definición comúnmente aceptada, una «acción colectiva» está compuesta por un conjunto de individuos o actores que comparten un cierto interés, objetivo o necesidad, el resultado de la cual es un cierto producto denominado «bien» y una cierta forma de distribuirlo, puesto que no necesariamente será consumido por los que han participado, sino que beneficiará también a otros.

En el proceso de diseño de una política de acomodación se producen muchos niveles de acción colectiva. Según las premisas introducidas anteriormente, éstos corresponden a la dimensión institucional. Contemplamos así dos posibles niveles:

Un primer nivel corresponde a la dimensión subjetiva institucional. Está compuesto por cuatro tipos de actores: las asociaciones de inmigrantes y las ONGs que trabajan el tema, los partidos políticos, las organizaciones de intereses y la administración pública (en distintos niveles: local, autonómico, estatal). 
Un segundo nivel correspondiente a la dimensión objetiva institucional. Existen acciones colectivas de acuerdo con la lógica de cada esfera. En este nivel contemplamos ocho esferas estructurales: convivencia social, empadronamiento, vivienda, trabajo, sanidad, educación, seguridad y cultura.

\section{B. Bienes primarios}

Entiendo por «bien primario» todo recurso que el inmigrante considera como necesario para poder ser autónomo y miembro plenamente acomodado de la sociedad. Las dos características principales del bien primario son que es instrumental, puesto que especifica los medios necesarios, y constituye el principal objeto reivindicativo del inmigrante para poder ser autónomo ${ }^{16}$. Asimismo, incorporo el enfoque histórico y antropológico de la teoría de bienes de M. WALZER. Estos bienes (recursos) poseen al menos dos características principales: (i) son bienes sociales, es decir, están socialmente constituidos, y (ii) son bienes locales, en tanto que cada uno configura una esfera distributiva con una lógica interna propia en la cual sólo unos determinados principios de justicia pueden desempenar la función reguladora que se les asigna ( $M$. WALZER, 1983: 7-10). Estas dos características principales apoyan la conclusión normativa de que bienes distintos deberian ser distribuidos por razones distintas, en arreglo a diferentes procedimientos y por distintos actores, y que todas estas diferencias derivan de la comprensión de los bienes mismos, lo cual es producto inevitable del particularismo bistórico y cultural (M. WALZER, 1983: 6). En síntesis, que todo diseño de una política de acomodación está orientada por una noción de justicia local.

Contemplamos dos tipos de bienes primarios, según sean considerados como estructurales (o pertenecientes a cada una de las esferas de la estructura básica de la sociedad) o mediadores (o que sirven al inmigrante para ser autónomo en una o más de una esfera de la estructura básica). Como en el caso de los actores que intervienen en la acción colectiva, los bienes estructurales han sido determinados previamente: son los que conforman cada esfera estructural: cada sector es considerado como distribuyendo un bien deseado por el inmigrante para poder ser autónomo. El segundo tipo, los bienes mediadores, puede ser de dos tipos: bienes mediadores comunitarios (nacionalidad, cultura, religión) o individuales (edad, género, situación jurídica, situación familiar y de parentesco, permiso de residencia, permiso de trabajo, profesión, habilidades físicas, formación académica y nivel estudios, conocimiento de la lengua autóctona).

El objetivo de una política de acomodación será, pues, llegar a un «equilibrio reflexivo» entre los bienes estructurales que requiere el inmigrante para ser miembro pleno de la sociedad, y los bienes mediadores que precisa para llevar a cabo una vida autónoma ${ }^{17}$.
Los bienes estructurales poseen unas propiedades que determinan el potencial conflictivo que se puede generar en el proceso de acción colectiva (relacionamos aquí los componentes A y B del marco analítico). Cuando hablo de conflicto significa que no hay un consentimiento predeterminado en la forma de diseñar una política de acomodación y de decidir si se debe cambiar o no la estructura básica, y si esa modificación, si se resuelve como necesaria, será débil o fuerte. Es en la resolución de estos conflictos donde intervienen los tres modelos de acomodación mencionados anteriormente: los modelos asimilacionista, integracionista, y autonómico.

Según las propiedades de cada bien podemos, pues, localizar donde radica su potencial conflictivo para elaborar una política de acomodación. Esta modificación puede ser débil o fuerte. La diferencia fundamental estriba en que la débil es el resultado de una decisión administrativa, mientras que la fuerte es producto de una decisión politica. Será también débil si, y sólo si, afecta a unos actores estructurales determinados en la acción colectiva, pero no a todos. Por ejemplo, el hecho de que un administrador tenga que comunicar con un inmigrante en su propio idioma, que se difundan informaciones oficiales en lenguas propias de los inmigrantes, que un maestro opte por variar el contenido de su asignatura para acomodar a niños inmigrantes o que un médico deba cambiar su forma de detectar síntomas de enfermedad por tratarse de una mujer magrebí. En cambio, si la modificación de la estructura básica es fuerte afectará a más de una esfera y a más de unos actores estructurales. Por ejemplo, el hecho de tener que variar la distribución del cementerio para enterrar a inmigrantes magrebíes; el hecho de tener que modificar el currículum educativo de los escolares en general. En síntesis, las variaciones débiles son internas a una esfera estructural y/o dependen de la libre voluntad (la buena disposición) de los actores implicados, y las fuertes afectan externamente a más de una esfera y/o no dependen directamente de la voluntad del actor implicado, sino que proceden de una decisión política. Se sigue que las modificaciones fuertes tendrán un potencial conflictivo más elevado que las débiles.

Considero que existen cuatro tipos de propiedades, presentadas cada una por relaciones binarias ${ }^{18}$. En su descripción he localizado su potencial conflictivo para consensuar una politica de acomodación. Si bien al considerar los bienes estructurales nuestra referencia ha sido la del inmigrante como receptor de beneficios, el potencial conflictivo se determina considerando el grado de modificación o no que requieren las diferentes esferas que conforman la estructura básica para que los inmigrantes sean en ellas autónomos. Para discriminar su virtual conflictividad, cambio momentáneamente, pues, de punto focal, y me sitúo desde el punto de vista del «acomodador», esto es, la sociedad receptora ${ }^{19}$. 


\section{Escasez/abundancia del bien}

Un bien es escaso cuando no hay suficiente para sausfacer a todos los inmigrantes que lo desean o necesitan para ser autónomos. Su potencial para el conflicio es fuerie puesto que se supone que habrá más inmigrantes que quieran beneficiarse del bien que ofertas.

\section{Homogeneidad/heterogeneidad}

Para entender esta dicotomía debemos situarnos desde el punto de vista del valor genérico de un bien y compararlo con sus unidades concretas. Si todas las unidades poseen igual ator para cuatquier aeter, al menos en los aspectos que lo bacen necesario, el bien es homogéneo; si no poseen igual valor, el bien es heterogéneo. En este último caso, puede suceder que para un actor algunas unidades del bien pueden ser consideradas como siendo de mejor calidad que otras, y, por lo tanto, puede interpretar que son más intensamente deseadas por los inmigrantes para acomodarse que otras. Esto es, y en términos comparativos, puede ocurrir que para unos actores algunas unidades del bien sean superiores, y para otros estas mismas unidades sean inferiores. Vemos que la diferencia entre ambas propiedades es bastante fluctuante. Depende en último término de la percepción subjetiva del actor envuelto en una acción colectiva ${ }^{20}$. El potencial de conflicto será fuerte en los bienes heterogéneos.

\section{Material/simbólico}

Un bien es material cuando satisface directamente las necesidades físicas del inmigrante, mientras que será simbólico en otros casos de necesidades. Desde el punto de vista de la sociedad receptora, los bienes simbólicos tendrán un potencial conflictivo fuerte, puesto que le exigirán modificar ciertos aspectos «culturales» de su estructura básica.

Individual/colectivo ${ }^{21}$

Un bien es individual si pierde gradualmente valor al incrementarse su uso compartido. Será colectivo en caso contrario, esto es, que el bien no pierde valor conforme aumenta su uso compartido. Se sigue que el potencial conflictivo para diseñar una política de acomodación será fuerte en propiedades de bienes colectivos, puesto que la entrada de inmigrantes en el beneficio de este bien puede ser interpretado por algunos actores como una pérdida de su valor colectivo originario. Esto es, se exigirá una modificación fuerte de la estructura básica para continuar asegurando la propiedad colectiva dél bien con la entrada de nuevoś receptores, los inmigrantes.

Podemos resumir el potencial conflictivo de cada propiedad de bien de la forma siguiente.

\begin{tabular}{|c|c|}
\hline$\therefore$ Potencial conflictivo fuerte & Potencial conflictivo débil \\
\hline $\begin{array}{c}\text { Escaso } \\
\text { Heterogéneo } \\
\text { Simbólico } \\
\text { Colectivo }\end{array}$ & $\begin{array}{c}\text { Abundante } \\
\text { Homogéneo } \\
\text { Material } \\
\text { Individual }\end{array}$ \\
\hline
\end{tabular}

Asimismo, aplicando las distinciones efectuadas a los tipos de bienes estructurales que consideramos en este estudio, podemos obtener una tabla de doble entrada ${ }^{22}$.

\begin{tabular}{|c|c|c|c|c|}
\hline & Escaso & Homogéneo & Material & Individual \\
\hline Convivencia & No & Si & No & No \\
\hline Empadronamiento & No & Si & No & No \\
\hline Vivienda & Si & SiNo & Sí & Sí \\
\hline Trabajo & Sí & Sino & Sí & $\mathrm{Si}$ \\
\hline Sanidad & No & $\mathrm{Si}$ & Sí & No \\
\hline Educación & No & Sino & No & No \\
\hline Seguridad & No & Sí & $\mathrm{Si}$ & No \\
\hline Cultura & No & Sí & No & No \\
\hline
\end{tabular}

Finalmente, correlacionando los dos cuadros anteriores, podemos avanzar como hipótesis la(s) propiedad(es) distinti$\mathrm{va}(\mathrm{s})$ del bien que le hacen fuertemente conflictivo para elaborar una política de acomodación. Podemos establecer 4 grupos (entre paréntesis ponemos aquellas propiedades de bien que pueden ser objeto de discusión según la percepción subjetiva que pueda tener un actor concreto):

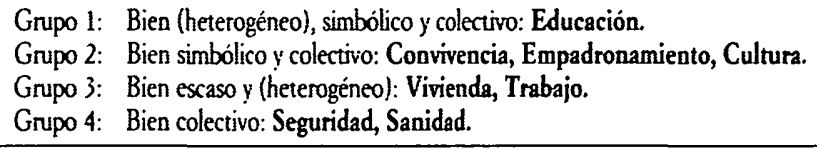

Según las propiedades del bien, los que poseen un potencial fuerte de conflictividad son, por orden, la educación; la vivienda y el trabajo; la convivencia social, el empadronamiento y la cultura; la sanidad y la seguridad.

Aunque estos datos nos proporcionan cierta información sobre el tipo de política de acomodación requerida de acuerdo con la especificidad de las propiedades de los bienes estructurales, no nos podemos aventurar a emitir conclusiones precipitadas. Anunciamos, no obstante, que el análisis de las entrevistas nos ha confirmado que las lógicas argumentales de los actores se centran para cada esfera en estas propiedades. Las otras, al no ser consideradas como conflictivas fuertes, apenas han recibido una atención prioritaria en los razonamientos de los actores ${ }^{23}$. Para completar nuestro marco analítico necesitamos todavía localizar los bienes mediadores (individuales y/o comunitarios) que intervienen en cada esfera. Éstos son los que circunscribirán la forma que deberá tomar la modificación de la estructura básica institucional.

\section{Principios de justicia}

Cuando hablo de justicia, no me refiero al hecho de si es justo o no el no haber nacido ciudadano en un determinado país, o el haber nacido pobre o en una sociedad del tercer mundo, sino que la administración de la que dependo distribuya de tal modo los bienes que no me proporcione recursos suficientes para salir de esta situación inicial. La justicia e injusticia hacen referencia, pues, al qué y al cómo los bienes se asignan. Parto de la base de que toda política de acomodación está orientada por unos principios y criterios de justicia, cuya finalidad básica es proporcionar bienes estructurales a los inmigrantes para que ellos mismos puedan salir, manteniendo sus bienes mediadores, de la situación de desigualdad en la que se encuentran.

Asimismo, y de acuerdo con mi objeto de estudio, cuando hablo de justicia e injusticia tengo como línea de base para 
comparar la situación de los autóctonos o ciudadanos. Si la situación es la misma, es decir, que los inmigrantes tienen los mismos bienes estructurales que los autóctonos y que no precisan bienes mediadores distintivos para ser autónomos, entonces no existe injusticia. Mi enfoque se centra, pues, en las diferencias entre la situación del inmigrante y la del ciudadano ${ }^{24}$.

Una vez contextualizado el uso que haré de la noción de justicia, es preciso adentrarse en algunas distinciones analíticas importantes. Diferencio, así, entre principios y criterios de justicia. El principio contesta a la pregunta del cómo se asigna un bien según ciertos bienes mediadores del inmigrante. Estos bienes mediadores del inmigrante los denomino criterios. Por ejemplo, la nacionalidad es un criterio, la asignación de un bien $X$ según la nacionalidad $Y$ es un principio.

Contemplo así una correlación entre dos tipos de principios de justicia (presentados en relaciones binarias) en relación con los bienes mediadores ${ }^{25}$.

\section{Tipo 1: Principios específicos y principios genéricos}

Principios que hacen referencia a algunos de los bienes mediadores de los inmigrantes (principios especificos) y otros principios que no lo hacen (principios genéricos).

\section{Tipo 2: Principios de grupo y principios individuales}

Principios que invocan esencialmente las relaciones del inmigrante con otras personas de acuerdo con su pertenencia comunitaria (principios de grupo), y principios que se concentran exclusivamente en los bienes mediadores individuales de los inmigrantes (principios individuales). Por ejemplo, la subvención económica para construir mezquitas o locales para asociaciones de inmigrantes de una determinada nacionalidad serían del primer tipo, mientras que la formación profesional del inmigrante o su nivel de conocimiento de la lengua española (o de las otras tres lenguas oficiales del Estado) serían ejemplos del segundo tipo.

Damos a continuación una lista de criterios susceptibles de usarse para aplicar un principio según los bienes mediadores del inmigrante.

Edad - Género - Nacionalidad - Situación jurídica (regular o irregular) - Situación familiar y de parentesco (casado o no) - Permiso de residencia (tipo) Permiso de trabajo (tipo) - Profesión - Religión Habilidades físicas - Formación académica y nivel estudios (alfabetización, nivel escolar, diplomado, universitaria, etc.) - Conocimiento de la lengua autóctona.

Estos criterios pueden ser utilizados de una forma variable si los combinamos con los tipos de principios. Por lo tanto, según sean usados darán lugar a un tipo de principio u otro. La clasificación es la siguiente:

\begin{tabular}{|c|c|c|c|c|}
\hline \multirow[t]{2}{*}{ Tipo 1} & \multirow{2}{*}{$\begin{array}{l}\text { Principio } \\
\text { específico }\end{array}$} & & \multirow{2}{*}{$\frac{\text { de grupo }}{\text { individual }}$} & \multirow[b]{2}{*}{$\begin{array}{l}\text { Nacionalidad - Religión. } \\
\text { Edad - Género - Situación jurí- } \\
\text { dica - Situación familiar y de } \\
\text { parentesco - Permiso de residencia } \\
\text { - Permiso de trabajo - Profesión } \\
\text { - Habilidades físicas - Formación } \\
\text { académica y nivel estudios - Cono- } \\
\text { cimiento de la lengua autóctona. }\end{array}$} \\
\hline & & & & \\
\hline \multirow[t]{2}{*}{ Tipo 2} & Principio & a) & Puro & [ningún bien mediador] \\
\hline & & b) & $\begin{array}{l}\text { De acción } \\
\text { específica }\end{array}$ & $\begin{array}{l}\text { Edad - Género - Nacionalidad } \\
\text { - Situación juridica - Situación } \\
\text { familiar y de parentesco - Permiso } \\
\text { de residencia - Permiso de trabajo } \\
\text { - Profesión - Religión - Habi- }- \text { - } \\
\text { lidades físicas - Formación acadé- } \\
\text { mica y nivel estudios - Conoci- } \\
\text { miento de la lengua autóctona. }\end{array}$ \\
\hline
\end{tabular}

En las entrevistas realizadas se ha detectado una gran controversia para orientar políticas de acomodación sobre la pertinencia o no de usar criterios de acuerdo con los bienes mediadores distintivos de los inmigrantes. Pueden destacarse tres posiciones, cada una de ellas relacionada con un modelo de acomodación ${ }^{26}$. La que aboga para que desaparezca cualquier criterio que oriente una política de acomodación: procedería como si se desconocieran los bienes mediadores distintivos del inmigrante. Serian las que defenderían principios genéricos puros [Tipo 2.a)] y un modelo asimilacionista. La que aboga que los criterios del tipo de bienes mediadores deben tener una representación en cualquier política de acomodación pero manteniendo la orientación genérica [Tipo $2 . b$ ), principios genéricos de acción especifica], estarían orientados por un modelo integracionista. Y, por último, la que defiende que los criterios del tipo de bienes mediadores deben tener una representación en cualquier política de acomodación, abogaría por un modelo autonómico. Este modelo podría enfocarse en los bienes mediadores comunitarios [Tipo 1.a), principios especificos de grupo] o individuales [Tipo 1.b), principios especificos individuales].

Por último, contemplo dos posibles consecuencias al implementar un principio ${ }^{27}$. La primera sería la normal, y se denomina consecuencia primaria. Al aplicarse una política de acomodación siguiendo unos principios de justicia determinados, los efectos de dicha política son los esperados. Otra consecuencia es la que denominaré como efecto de exclusión. Al incluir a ciertas personas en el beneficio del bien, se excluye, como efecto secundario, a otras.

\section{Estructura básica institucional}

En primer lugar, necesitamos concretar qué significa estructura básica institucional, la que, según nuestro enfoque, se ve directamente afectada por la presencia de inmigrantes. 
Entiendo por estructura básica institucional ${ }^{28}$ a las principales instituciones políticas y públicas que operan en distintos niveles administrativos ${ }^{29}$. La función que juega en el sistema que estamos trazando es que opera como restricción en el proceso de diseño de una política de acomodación (sus acciones colectivas, el tipo de bienes que se asignan y los principios de justicia y modelos que se siguen) ${ }^{30}$.

Centrándonos en estos límites, contemplamos cuatro categorías básicas.

\begin{tabular}{|ll|}
\hline Categoria 1. & Límites estructurales. \\
Categoria 2. & Límites políticos. \\
Categoria 3. & Límites culturales. \\
Categoria 4. & Límites mediáticos. \\
\hline
\end{tabular}

Déjenme explicar cada una de estas categorías por separado. Aunque, insisto, en todo proceso de planificación de políticas de acomodación estos cuatro límites se interrelacionan constantemente. La división que hago es simplemente analítica.

\section{Categoría 1}

\section{Límites estructurales}

Son límites propios de nuestro sistema liberal democrático. Actúan como restricciones externas en el proceso de elaboración de políticas de acomodación. Dentro de esta categoría contemplamos dos subcategorias: los límites estructurales macro y micro.

\section{Límites macro}

Son aquellos que afectan a toda la población, sin distincion entre inmigrantes y ciudadanos. Son los estrictamente estructurales entendidos en sentido clásico: por ejemplo, la lógica del mercado, el desempleo, las políticas procedentes de la Unión Europea, la distribución territorial de competencias, etc.

\section{Límites micro}

Son los que afectan especialmente a la población inmigrante. Destacamos principalmente todo lo referente a la legislación y al sistema de derechos y de deberes que el Estado español destina a los inmigrantes, una vez han pasado regularmente la «puerta de acceso». Este nivel micro limita, por lo tanto, el espacio de acción legalmente reconocido para que el inmigrante sea autónomo. Es decir, existen determinadas acciones que el ciudadano puede hacer legalmente y que el inmigrante, por el solo hecho de serlo, no puede hacer sin incurrir en la ilegalidad. Por ejemplo, los inmigrantes no pueden votar, ni acceder a todas las esferas públicas sin excepción. Para los gestores públicos estos límites no pueden obviarse, puesto que también para ellos actúan como restricción para diseñar políticas de acomodación. En nuestros términos, estos límites son susceptibles de cambio por propia voluntad del Gobierno. Por ejemplo, el hecho de legislar una nueva regulación de Ley de extranjería, o de modificar la misma legislación orgánica, tiene unas consecuencias directas sobre el espacio que deja tanto al inmigrante para actuar en la esfera pública, como al gestor público para disenar políticas concretas. Los actores expresan una convicción generalizada de que estos límites micro son de capital importancia, puesto que actúan como restricciones externas para planificar estrategias de acomodación.

\section{Categoría 2}

Limites políticos

A diferencia de los anteriores, especialmente los límites micro (legislación), los límites políticos son estrictamente casos típicos de justicia local. Por lo tanto, son propias restricciones intermas para elaborar políticas de acomodación. Están determinados por las valoraciones y las concepciones concretas que tienen los actores encargados de elaborar políticas en una esfera pública determinada (el trabajo, la vivienda, la educación, por ejemplo). Tienen, además, y nuevamente a diferencia de las categorías anteriores, un carácter mucho más dinámico, en tanto que pueden variar por razones coyunturales. Aunque la diferencia es bastante difícil de desligar en la práctica, nos referimos a dos tipos de politicas: las políticas regulativas y las politicas distributivas. Según nuestro objeto de estudio, las primeras regulan el espacio de acción del inmigrante, y las políticas distributivas tienen como objetivo asignarle bienes estructurales (como la salud, la seguridad). Por ejemplo, según la concepción que tenga de la inmigración, un gestor público se inclinará más por un tipo determinado de política de acomodación en lugar de otro, dentro del espacio legal que tiene (es decir, si seguimos bien, respetando los límites estructurales micro). Insisto en que estas concepciones, además de tener un carácter dinámico, son locales en el doble sentido que le hemos dado, a saber, que dependen del grado de competencia que tenga la administración encargada, y del tipo de esfera estructural. En un nivel aplicado, lo que interesa subrayar es que tienen una función determinante para diseñar políticas de acomodación, en tanto que orientan al mismo tiempo acciones individuales y de grupo, y ciertas actitudes sociales. Dada la dimensión normativa del estudio, en las entrevistas se pueden contemplar principalmente limites evaluativos (expectativas sobre las políticas y los canales del sistema de acomodación existente, y de los actores implicados en las acciones colectivas); aunque también se pueden tener en cuenta, para limitar el análisis cualitativo, los límites cognitivos (grado de conocimiento sobre las políticas y canales del sistema de acomodación existente, y de los actores implicados en las acciones colectivas).

\section{Categoría 3}

Límites culturales

Se refieren principalmente a los mecanismos de socialización que tiene la estructura básica y que afectan de forma directa el diseño de políticas concretas de acomodación. Se trata específicamente de todo el sistema de prejuicios o preconcepciones culturales que orienta inevitablemente a todo actor al involucrarse en una acción colectiva. Asimismo, esta tercera categoría destaca que partimos de la premisa que la estructura institucional no es culturalmente neutra. Tiene mecanismos propios para garantizar que en la distribución de bienes se asignen al mismo tiempo pautas de conducta de carácter cultural. Entre los límites culturales más importantes, el marco analítico contempla dos indicadores: por un lado, los estereotipos, los cuales tienen, sin lugar a dudas, una influencia determinante en el momento de decidirse por un tipo u otro de acción estratégica; por otro lado, la figura emergente de los mediadores culturales, cada vez más visibles, aunque continúan conservando un carácter controvertido ${ }^{31}$. 


\section{Categoria 4}

\section{Límites mediáticos}

La estructura básica también limita la planificación de políticas de acomodación a través de uno de sus principales mediadores: los medios de comunicación. Los discursos y la forma de enfocar los temas relacionados con la inmigración tienen una influencia determinante no solamente en su función socializadora, sino también en su función legitimadora, cuando los gestores políticos deben decidirse por un tipo u otro de políticas de acomodación. En esta categoría incluimos, pues, las percepciones de los actores sobre el tipo de influencia que tienen los medios de comunicación en general para facilitar o no la acomodación de los inmigrantes.

\section{Contextos potenciales para futuras investigaciones}

El marco analítico propuesto ha sido obtenido metodológicamente combinando el razonamiento deductivo y la inferencia a partir de los datos del estudio citado. Aunque la base potencial está dada, para poder comprobar adecuadamente su viabilidad se requieren, no obstante, otras aplicaciones en contextos diferentes. De ahí que quisiera acabar insistiendo en que esta concepción compleja de la acomodación y el marco analítico propuesto debe conservar un carácter dinámico y abierto. Según el caso de estudio necesitaría sin duda ampliar y profundizar sus esquemas y categorías. Queda, asimismo, claro que el análisis de cómo gestionar la presencia de los inmigrantes no sólo debe tener en cuenta el carácter multidisciplinario del tema, sino también que es, desde el punto de vista de la estructura básica institucional, un fenómeno multinivel (afecta a todos los niveles de los poderes públicos) y multiactor (implica a un numeroso grupo de actores, no sólo administrativos y políticos, sino también procedentes de la sociedad civil y del mundo asociativo en general). En definitiva, que el sistema de acomodación es un sistema pluralista en todos los sentidos (políticos, administrativos y político-públicos).

Existen muchos temas teóricos y contextos donde la relación entre la ciudadanía y la interculturalidad es polémica ( $R$. ZAPA.
TA-BARRERO, coord.: 2001a). Este marco analítico podría servir de base de referencia para aplicarlo en numerosos contextos pendientes de analizar. A continuación enumero algunos, sin pretensión de exhaustividad, sino tan sólo indicativo:

1. En el contexto local de los Ayuntamientos directamente necesitados de un marco de referencia para orientar sus políticas de acomodación. De hecho, el marco propuesto ya ha sido objeto de aplicación en el contexto de la ciudad de Barcelona (investigación financiada por la Fundació Jaume Bofill), cuyos resultados finales están bajo forma de libro (R. ZAPATA-BARRERO, 2001d).

2. En el contexto de una Comunidad Autónoma directamente afectada por el tema de la inmigración. Una perspectiva comparada podría centrarse en las relaciones Comunidad Autónoma/Diputación (cuando exista)/Administración municipal.

3. En el contexto estatal. Por ejemplo el Estado español presenta una estructura política y administrativa descentralizada. En términos de poderes públicos las decisiones que se toman en materia de inmigración deben ser el resultado de esta estructura multinivel. Las relaciones entre las diferentes administraciones desde una perspectiva intergubernamental $e$ intersectorial podrían ser un punto de partida para analizar el modo en que las instituciones deberían organizarse entre ellas para acomodar a los inmigrantes. Se podría contemplar dos niveles de análisis. El nivel vertical (relación Comunidades Autónomas/Administración central), y el nivel horizontal (relaciones entre Comunidades Autónomas entre sí) ${ }^{32}$.

4. En el contexto de la Unión Europea. Por ejemplo, partiendo de las instituciones de la UE, podría aplicarse el marco desde una perspectiva inter-estatal, tomando como estudio de caso, por ejemplo, las relaciones entre la UE y el Estado español ${ }^{33}$.

Seguro que futuras investigaciones podrán aportar nuevas luces ante un hecho que acaba de adquirir la dimensión social y política que todos conocemos. La agenda política nos pide con insistencia propuestas serias y realistas ante un fenómeno en el cual los términos reto y complejidad son mayúsculos. Toda otra propuesta que avance soluciones y fórmulas milagrosas debe abordarse con cautela. El populismo y la demagogia científica e intelectual que despierta el fenómeno no resuelve problemas, sino que forman parte del problema a analizar. 
* En el proceso de elaboración final de este artículo me he beneficiado de los comentarios y críticas de los relatores y asistentes del panel del 18th IPSA World Congress donde defendi un paper titulado Three models of multicultural accommodation: An analytical framework for analysing immigrant's accommodation policies (Québec, 1-5 de agosto de 2000), y del II Congreso sobre la Lnmigración en España (Madrid, 5-7 de octubre de 2000).

* Profesor Titular de Ciencia Politica, Grup de Recerca de Teoria Politica, Universitat Pompeu Fabra (Barcelona).

' Véanse las referencias bibliográficas españolas y del debate internacional en $R$. ZAPATA-BARRERO (2000d).

${ }^{2}$ Se da por supuesto que la inmigración de la que tratamos es la extra-comunitaria procedente principalmente de paises en vías de desarrollo.

${ }^{3}$ El primer nivel, el del acceso, ya fue objeto de análisis para el caso español en R. Zapata-BarRero (2000b). También abordé el tema, junto con el nivel de la coexistencia, en R. Zapata-Barrero (2000d, e).

${ }^{4}$ Se trata de un estudio financiado por la Fundació Jaume Bofill (finalizado en marzo de 2000) cuyos resultados han aparecido en R ZapaTA-BARRERo (2001d).

'Este término tiene una extensa y controvertida literatura que ya ha sido objeto de estudio en algunos de mis trabajos anteriores (R. ZAPATA-BARRERO, 1999, 2001b). En general, por «autonomia» entenderé la situación en la que se encuentra el inmigrante de poder decidir sus planes de vida y/o los de su grupo, y de poder practicarlos públicamente con independencia de los límites específicos que le impone la estructura básica institucional en general y su situación legal en particular.

${ }^{6}$ Agradezco a J. Jordana por familiarizarme con esta literatura y sugerirme vías para aplicarla en mi caso de estudio.

${ }^{7}$ Usaré indistintamente «esferas públicas», «esferas locales» y «esferas estructurales».

${ }^{8}$ Merece la pena advertir que en el tratamiento de la politica de inmigración MACKIE vulnera una de sus premisas metodológicas, la de que las instituciones que se encargan de su asignación son en mayor o menor grado independientes del gobierno. Tal como trata la política de inmigración, y siguiendo esta variable, sería más bien un caso de Justicia Global.

9 Insisto en que es evidente que la primera afecta a la segunda. En las entrevistas realizadas en el estudio citado, la respuesta a la pregunta sobre si el ritmo y el contenido del desarrollo legislativo en España afecta la integración de los inmigrantes fue prácticamente afirmativa de forma unánime.

${ }^{10}$ Esta diferencia es importante. En efecto, EISTER (1994: 38) se ocupa de políticas distributivas, en presencia sobre todo de bienes escasos. Los bienes abundantes no son considerados como objeto de interés para una justicia local, como en este caso.

"Para ampliar la concepción que tengo de la integración, véase R. ZAPATA-BARRERO $(2000 \mathrm{~g}$, h.).

${ }^{12}$ Véase el útil material de consulta de G. MalGESinI y C. GimÉnez (1997), así como de J. Rex (1996).

${ }^{13}$ Antes de presentarlos conviene hacer tres precisiones importantes para evitar equivocos sobre la función que tienen en el marco analítico: (i) Tienen un carácter estrictamente analítico: los tres modelos que contemplamos sirven de base para orientar politicas públicas, pero no dan información sobre cómo implementarlas. En este apartado doy, pues, solamente sus límites externos. En términos jurídicos diriamos que presentamos una Ley Orgánica pero sin su regulación, «un motor sin su gasolina». (ii) Tienen un carácter estrictamente independiente: aunque confieso que yo mismo al principio tuve esta tendencia, estos tres modelos no deben tomarse de forma evolutiva, como si el modelo siguiente implicara el anterior. (iii) Tienen un carácter descriptivo: aunque confieso nuevamente que tuve esta predisposición al principio, cada uno de los tres modelos no corresponden necesariamente a una concepción normativa determinada. Por lo tanto, ni el modelo asimilacionista corresponde a la concepción neoliberal o libertaria, ni el integracionista a la liberal, ni el autonómico a la democrática.
14 Véanse, entre otros, Ch. KuKatuas (1992) y J. Gray (1993).

is Sobre el concepto de reconocimiento, véase, por ejemplo, Ch. TAYLoR (ed., 1992).

${ }^{16}$ Adapto defuniciones procedentes de J. Rawrs (1993: 223-4). Véase también R Zapata-BARRERo (2001b).

i7 Por «equilibrio reflexivo» entiendo la técnica propuesta por Ravrs que permite conectar la teoría y la práctica de la justicia (RAwrs, 1971: 37 ss., 68 ss., y 1993: 8). En general, el «equilibrio reflexivo» permite que el acuerdo alcanzado (en nuestro caso el resultado de una acción colectiva para determinar una política de acomodación) esté conforme con las convicciones y concepciones particulares de los inmigrantes, es decir, y en nuestro lenguaje, con sus bienes mediadores.

${ }^{18}$ Para las dos primeras me he basado en ELSTER (1994: 33-34; ed. 1995: 5), y para las dos siguientes en M. WARREN (1992: 17).

${ }^{19}$ Como comprobará el lector, parto del supuesto, ya aludido al precisar la pregunta básica del estudio en la introducción, que el que crea conflicto no es el inmigrante, sino la estructura básica de la sociedad que no tenía previsto tener como receptores a esta población recién llegada.

${ }^{20}$ ELSTER da como ejemplo el servicio militar (1994: 34). Si el bien se percibe como un todo genérico no importando sus unidades, el bien es considerado como homogéneo. Ahora bien, para algunos beneficiarios puede ocurrir que le importen el destino territorial o el cuerpo militar. En este caso, el bien será considerado como heterogéneo.

${ }^{21} \mathrm{M}$. WARREN (1992: 17) habla de individual/social, optamos por utilizar «colectivo» para no confundir al lector, puesto que, como ya hemos subrayado, la naturaleza de los bienes que tratamos es inherentemente social.

${ }^{22} \mathrm{Si}$ ponemos Si/No significa que depende de la percepción del inmigrante en casos concretos. Por ejemplo, el trabajo puede ser considerado como homogéneo si lo que importa al inmigrante es trabajar independiente de sus preferencias laborales; mientras que será considerado como heterogéneo si lo que importa al inmigrante es el tipo de trabajo, es decir, que no trabajará a cualquier precio.

${ }^{23}$ Para ampliar información, véase R. Zapata-Barrero (2001d).

${ }^{24}$ Insisto en que me ocupo del modo en que las instituciones públicas asignan bienes estructurales. Los principios de justicia son aplicados por las instituciones públicas y no por las personas ni por las instituciones privadas (RAWLS, 1971: 54; ELSTER, 1994, ed. 1995). El lenguaje que uso es, pues, un lenguaje sobre juicios morales finales. La justicia establece los parámetros moralmente legítimos de una política pública (J. Carens, 1995: 1-17).

${ }^{25}$ Adapto a mi estudio a ELSTER (1994: 81-122).

${ }^{26}$ Me baso de nuevo en ELSTER (ed. 1995: 14), aunque adaptándolo a mi objeto de estudio.

${ }^{27}$ Adapto de nuevo a ELSTER (1994: 131-152).

${ }^{28}$ Usaré indistintamente «estructura básica», «estructura institucional» o una combinación de ambos, esto es, «estructura básica institucional».

${ }^{2}$ Adapto a J. RawLS (1993: 11) a mi objeto de estudio. Por continuar precisando conceptos, por «institución» significo básicamente un sistema de reglas públicamente reconocido que define cargos y posiciones con sus derechos y deberes, poderes e inmunidades (J. Rawls, 1971: 55), y por medio del cual se establecen unas expectativas mutuas entre las personas (A. HAMLIN y Ph. PETTT, 1989: 2). Lo que me interesa de esta definición es que las instituciones implican una cierta conducta, cuyos limites son o pueden ser conocidos. Una persona que forma parte de una institución conoce las reglas y sabe lo que les pide hacer a él y a los otros.

${ }^{30}$ Desde el punto de vista del sistema politico de acomodación, que es lo que nos interesa aqui subrayar, estas instituciones limitan la acciones colectivas (componente A) y los principios de justicia (componente C) para decidir la asignación de los bienes primarios (componente $\mathrm{B}$ ) a los inmigrantes.

"Algunos resultados de esta categoria se encuentran en R. ZAPATA-BARRERO (2000i).

${ }^{32}$ Éste es el contexto del actual grupo de trabajo que dirijo en el marco de una investigación que acaba de iniciarse a escala estatal, financiada por el Ministerio de Ciencia y Tecnologia, y titulada: Democracia avamada y políticas de integración de la inmigración: relaciones intergubernamentales y limites del Estado de Bienestar (SEC 2000: 534).

${ }^{33}$ Véanse algunos primeros intentos en R. Zapata-Barrero (1998, 2000a, 2001c). 


\section{Bibliografia}

CARENS, J. (1995), «Immigration, welfare and justice», en W. F. SCHWARTZ (ed.), Justice in immigration, Cambridge: Cambridge University Press: 1-17.

DAHL, R (1992), La democracia y sus críticos, Barcelona: Paidós.

ELSTER, J. (1985), «Rationality, morality and collective action», Ethics, 96: 136-155.

- (1990), «Local Justice», Archives Européennes de Sociologie, 31/1: 117-140.

- (1994), Justicia Local, Barcelona: Gedisa.

- (1995), Local justice in America, Nueva York: Russell Sage Foundation.

- (1995) (ed.), «Introduction: the idea of local justice», en (ed.): 1-24.

Gray, J. (1993), «The politics of cultural diversity», en Post-liberalism studies in political thought, Nueva York: Routledge: 253-71.

Hamun, A., y Pettr, Ph. (eds.) (1989), The good polity, Oxford: Basic Blackwell.

Hov, J, (1986), «Binary games as models of public goods», Scandinavian Political Studies, 9/4: 337-360.

KuKathas, Ch. (1993), «Are there any cultural rights?», Political Theory, 20/1: 105.139.

JORDANA, J., «Acción colectiva y asociaciones de intereses», Manuscrito inédito.

MACKIE, G. (1995), «U.S. Immigration policy and local justice», en J. ELSTER (ed.): $227-290$

MaLGesini, G., y Giménez, C. (1997), Guia de conceptos sobre migraciones, racismo $e$ interculturalidad, Madrid: La cueva del Oso.

Oliver, P. E. (1993), «Formal models of collective action», Annual Review of Sociology, 19: 271-300

Olson, M. (1965), The logic of collective action, Cambridge: Harvard University Press.

Rawls, J. (1971), A Theory of Justice, Cambridge: Harvard University Press.

- (1993), Political Liberalism, Nueva York: Columbia University Press.

REX, J. (1996), Ethnic minorities in the modem nation state, Londres: MacMillan.

TAYLOR, Ch. (1992), Multiculturalism and «the politics of recognitions, Princeton: Princeton University Press.

WALZER, M. (1983), Spheres of Justice, Nueva York: Basic Books.

WARREN, M. (1992), «Democratic theory and self-transformation», American Political Science Rev., 86/1: 8-23.
ZAPATA-BARRERO, R (1998), «Thinking about European citizenship from the perspective of a potential Euro-foreigners, en U. K. PrEuss y F. REQUEjo (eds.), European citizenship, multiculturalism and the State, Baden-Baden: Nomos: 31; cap. 9: 151-168.

- (1999), «¿Necesitamos un nuevo concepto de ciudadanía? Estabilidad democrática y pluralismo cultural», Revista Internacional de Filosofia Politica, 13: 119.149.

- (2000a), «Politica de inmigración y Unión Europea», Claves de la razón práctica, 104: 26.32 .

- (2000b), «Justicia para inmigrantes: mercado y política de extranjería», REIS, 90 (abril-junio): 159-181.

- $(2000 c)$, «Nous reptes per a la teoria liberal-democràtica: justícia i immigració», Diàlegs, vol. III, 8: 31.55 .

- (2000d), «Inmigración e innovación política», Revista Migraciones, 8.

- (2000e), «La época de la inmigración y su tratamiento politológico: una nota bibliográfica», Revista Española de Ciencia Política, 3: 167-180.

- (2000f), «Estats, nacions, i ciutadans en un entorn multicultural», Revista Ambits, 16-17: 49-54.

- (2000g), «La relació entre els immigrants i les Administracions: onze temes bàsics per a debatre politiques d'integració», Paper de la Fundació Rafael Campalans, 122.

- (2000h), «Ocho preguntas básicas para una cultura de la integración», La Vanguardia, 29 de octubre: $34-35$.

- (2000i), «Cultura, política e inmigración: estereotipos y mediadores cultura. les», Crítica, núm. 879 (noviembre): 29.33.

- (2001a) (coord.), Ciudadania e interculturalidad: balance y penpectivas para el siglo XXI (febrero-marzo, 19??) (contiene arts. de R. ZAPATA, F. REQUEJO, J. DE LuCas, V. CAmps, A. Rivero, J. M. Rosales y A. Giménez).

- (2001b), Ciudadania, democracia y pluralismo cultural: bacia un nuevo contrato social, Barcelona: Anthropos.

- (2001c), «The limits of a multinational Europe: democracy and immigration in the European Union», en F. ReQuejo (ed.), Democracy and national pluralism, Routledge.

- (2001d), L'bora dels immigrats: esferes de justicia i polítiques d'acomodació (título provisional), Barcelona: Centre d'Estudis de Temes Contemporanis (Col-lecció Temes Contemporanis/Proa). 